\title{
PRELIMINARY OBSERVATIONS AT IO-CM WAVELENGTH USING THE NRL 84-FOOT RADIO TELESCOPE
}

\author{
E. F. MCC LAIN AND R. M. SLOANAKER \\ Radio Astronomy Branch, U.S. Naval Research Laboratory \\ Washington, D.C., U.S.A.
}

The largest steerable radio telescope in the United States (Fig. 1) has recently been completed at the U.S. Naval Research Laboratory's Maryland Point Observatory in Charles County, Maryland. The new instrument, an 84-foot parabolic antenna, or "dish," was designed by the D. S. Kennedy Co. of Cohasset, Massachusetts. Originally ordered in June 1955, the instrument has been three years in design, construction, and erection. Carried on a polar or equatorial mount, the 84 -foot aluminum reflector can be aimed at any point in the sky and can track any celestial object from horizon to horizon. It is the largest antenna with this type of mounting in the world.

Structurally, the telescope (Fig. 2) consists of a hollow truncated steel cone approximately 40 feet high, at the top of which are mounted the polar and declination axes with their respective drive components. Steel truss work attached to the declination axis carries the aluminum reflector. The total weight of the instrument is about 169,000 pounds exclusive of 252 cubic yards of concrete in the foundation. The reflector itself, which weighs only 15,000 pounds, is made up of a tubular aluminum back-up truss work, to which is attached a surface of $\frac{8}{8}$-inch square aluminum mesh. The design specification called for a surface with a maximum deviation from a true parabola of $\pm \frac{8}{8}$ of an inch permitting operation to wavelengths as short as $10 \mathrm{~cm}$. To facilitate construction, the reflector was fabricated in eighty individual radial sections, each matched to a male mold. These sections were then accurately assembled in position by means of a precision theodolite survey.

The advantages of a polar mount are partially offset by the severe structural problem that arises in transferring the mechanical load of the dish to the supporting structure as the dish rotates about its own axis with respect to the earth. This mechanical problem has been effectively overcome in the Kennedy design with no sacrifice in sky coverage.

In addition to the sidereal or clock drive incorporated in the polar axis system, two other drive modes are incorporated in both the polar and declination axes. The first, a 15-degree per minute slew mode permits rapid motion from one point in the sky to another. The second, a so-called scan mode, permits any velocity from about \pm 0.1 to \pm 1 degree per minute to be imparted to either or both the declination and polar axes. The scan motions may be used while tracking at sidereal rate and are a great convenience in mapping and positional work. As may be seen from Fig. 3, no servos are employed in the drive system, and all drive motors are under the direct 




Fig. 1. 84-foot radio telescope at Maryland Point Observatory. 
control of the operator by means of keys at the control console. Polar and declination axis positions are displayed at the console by synchros driven from the respective axis. In addition, two hour-angle increment dials (degrees and minutes) indicate the condition of the polar-axis scan drive at all times. Horizon, polar axis, and north celestial pole limit switches are provided to prevent the operator from moving the telescope beyond design limits.

The location of the new observatory as shown in Fig. 4 is on Maryland State Route 224, five miles west of Riverside, Maryland, on the north shore of the Potomac River. This particular site was chosen after radio noise surveys had been conducted at a number of sites around the Washington area. The same equipment was employed as that used in the prelimi-

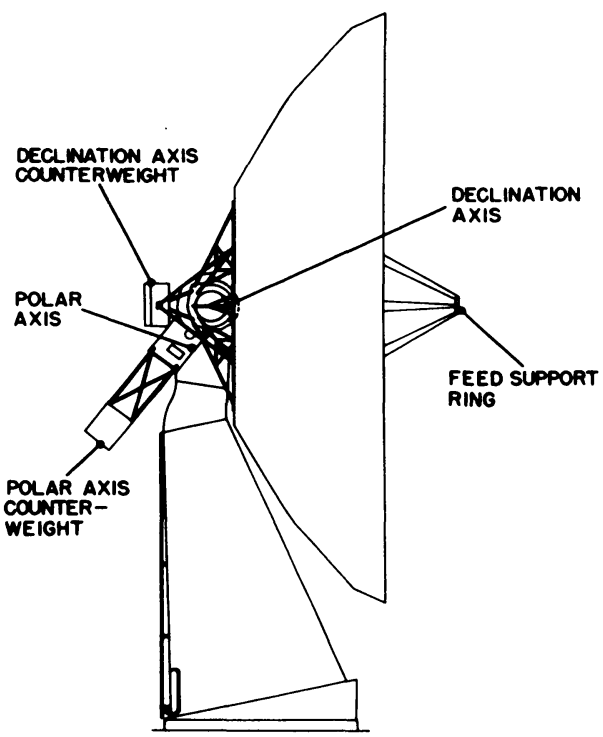

FIg. 2. Outline drawing of 84-foot antenna showing location of axes with respect to conical tower and mirror. Instrument is pointing at southern horizon. nary search for a national radio observatory site under the auspices of Associated Universities, Inc. The location at Maryland Point was by far the quietest site investigated within 50 miles of Washington and compared favorably with the third or fourth choice sites in
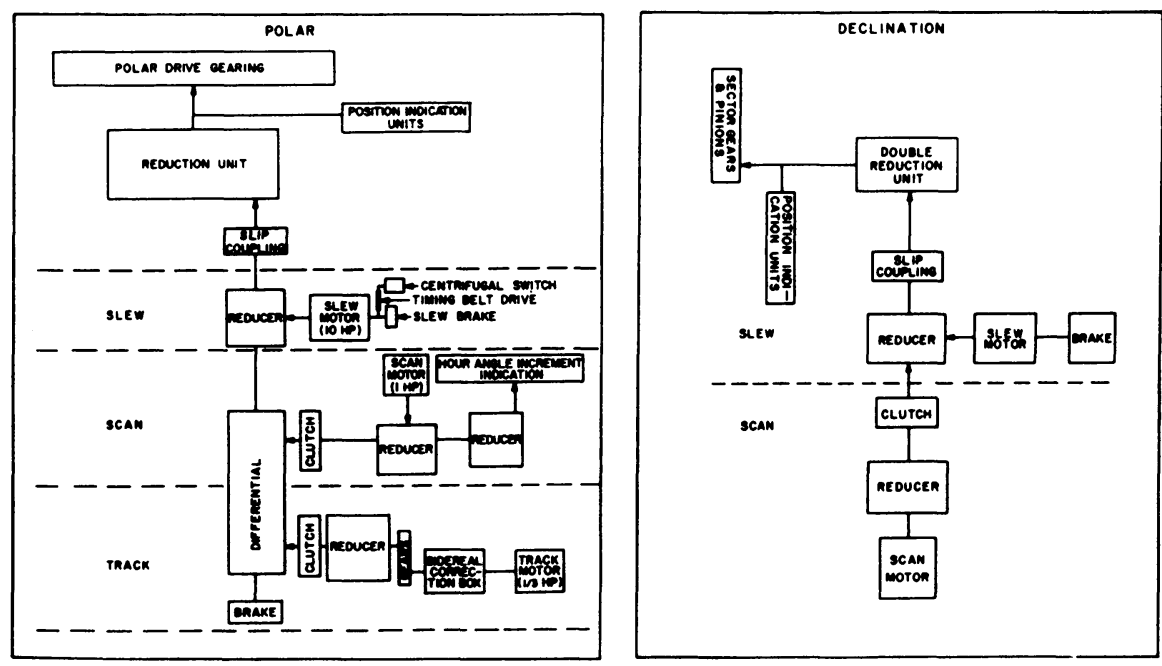

FIG. 3. Block diagram of polar axis and declination axis drive systems for 84 -foot antenna. 


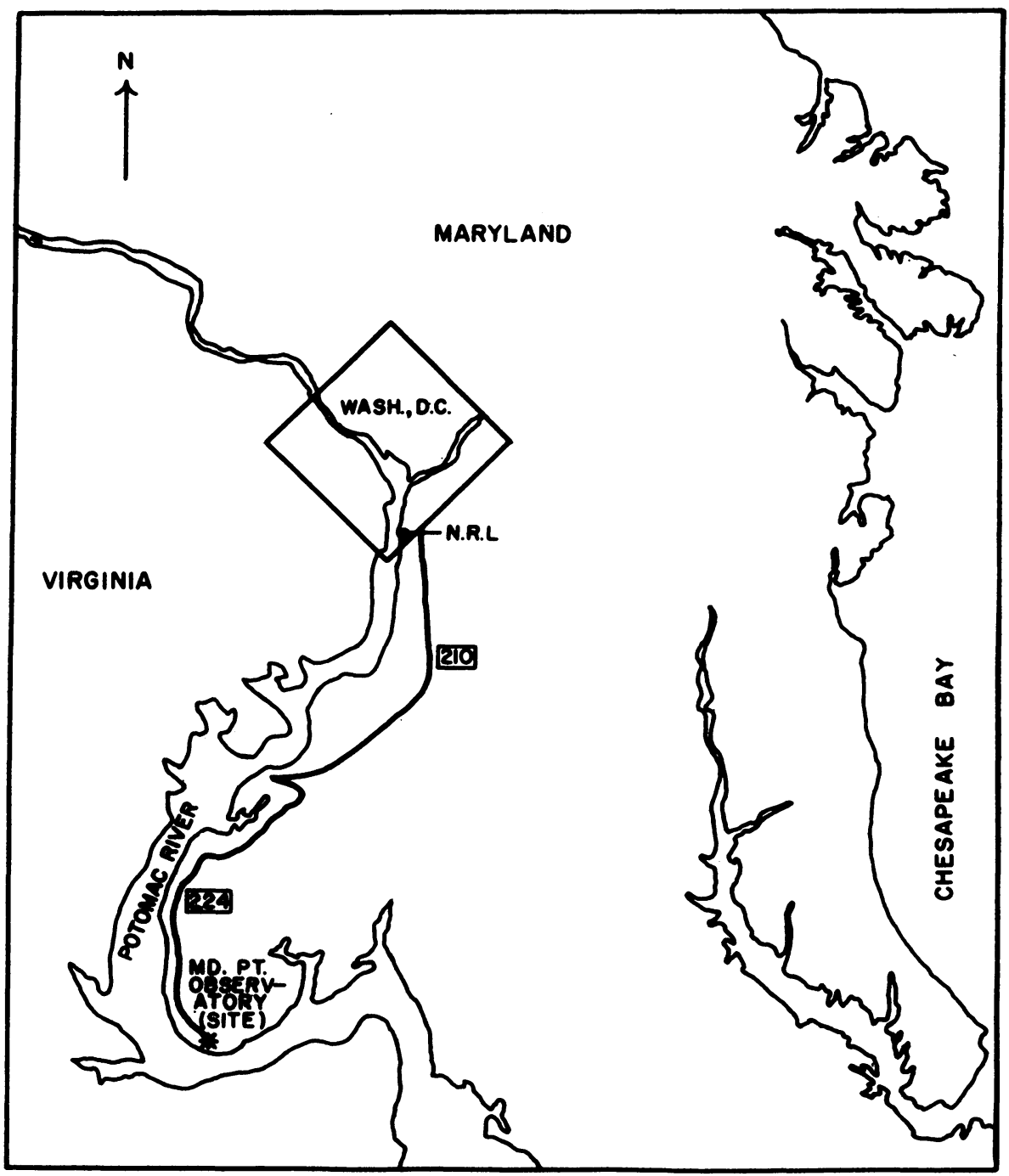

Fig. 4. Area map showing location of Maryland Point Observatory with respect to Washington, D.C.

the AUI survey. The specific location was chosen because of an unrestricted southern horizon. In our noise survey, as with the AUI survey, noise level seemed to correlate rather closely with population density. The site is approximately 45 miles from the Laboratory and requires about an hour's travel time by automobile.

The original specifications for the 84-foot instrument called for a surface accuracy of $\pm \frac{8}{8}$ inch and an absolute pointing accuracy of \pm 2 minutes of arc. To insure that these specifications were met, the dish was surveyed with a Wild T-1 theodolite following erection. Ninety check points at the 
panel corners were measured and, following adjustment, approximately ten of these test points approached zero error, approximately ten approached $\frac{8}{8}$-inch error, and the remainder were on the order of $\$$ inch. Following the final survey, a 5-inch camera was mounted in the polar-axis housing in such a manner that a field surrounding the pole star could be photographed. Two- and four-hour exposures were then taken with the instrument in the tracking mode to check polar-axis alignment. After the appropriate adjustments, polar-axis alignment was found to be better than 30 seconds of arc. A second camera was mounted on the declination axis, and multiple exposures taken at different declinations while tracking indicated that the polar axis and declination axis were perpendicular to within about 1 minute of arc.

Following the above mechanical tests, a new $10-\mathrm{cm}$ radiometer designed by $R$. M. Sloanaker was installed in the antenna. The radiometer is of the Dicke type [1] and employs noise injection as originally used by Drake and Ewen [2]. The data is calibrated at frequent intervals by using an argon gas discharge tube that was originally calibrated by thermal noise sources. The receiver accepts power in two frequency bands each $5.5 \mathrm{Mc} / \mathrm{s}$ wide separated by $60 \mathrm{Mc} / \mathrm{s}$ about a center frequency of $2910 \mathrm{Mc} / \mathrm{s}(10.3 \mathrm{~cm})$. Output time constants of $4^{\mathrm{s}}$ and $48^{\mathrm{s}}$ have been used. The rms fluctuation in the receiver output recorder trace is about $0.5^{\circ} \mathrm{C}$ for the $4^{\mathrm{s}}$ time constant, and about $0.15^{\circ} \mathrm{C}$ for the $48^{\mathrm{s}}$ time constant.

Using this radiometer, Sloanaker is making a survey of the brighter radio sources to permit final adjustment of the antenna, focal point, and mounting ring. This work is still in progress but indications are that the antenna will easily perform with an absolute pointing accuracy of \pm 2 minutes of arc, and it is hoped that with sufficient study of the systematic errors it will be possible to determine relative positions to within \pm 1 minute of arc. At $10.3 \mathrm{~cm}$, the aperture efficiency of the antenna is approximately 40 per cent. This figure is based on spectra of the brighter sources obtained from surveys

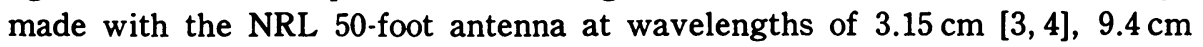
$[5,6]$, and $21 \mathrm{~cm}[7]$. The 40-per cent aperture efficiency agrees well with the result measured at the Bell Laboratories [8] for a $\lambda / 8$ surface. Drift curves and declination scans of Cassiopeia A and Cygnus $A$ indicate that the half-power beamwidth of the antenna at $10.3-\mathrm{cm}$ wavelength is 18.5 minutes of arc in right ascension ( $H$-plane pattern) and 17.5 minutes of arc in declination ( $E$-plane pattern), assuming that these sources produce no broadening; this result agrees well with the theoretical beamwidth of 17 minutes of arc. Fig. 5 is a drift curve of Cassiopeia A made at $10.3-\mathrm{cm}$ wavelength with a $4^{8}$ time constant. The measured antenna temperature of Cassiopeia $A$ is $93^{\circ} \mathrm{K}$ which corresponds to a flux density of unpolarized radiation for a point source $122 \times 10^{-25}$ watts $\mathrm{m}^{-2}(\mathrm{c} / \mathrm{s})^{-1}$. No antenna pattern measurements have been made as yet, but a study of the Cassiopeia $\mathrm{A}$ drift curves would indicate that the side lobes are not excessive.

Although the routine survey of the brighter sources is still in progress, two interesting findings have been made, which we feel should be reported at this time. First, radiation from Jupiter has been detected, and an analysis 


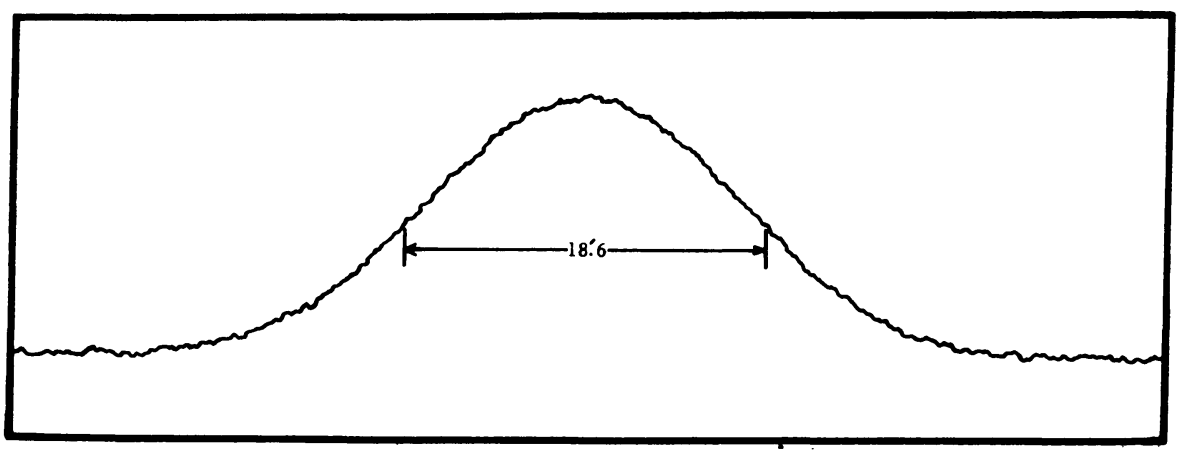

Fig. 5. Drift curve of Cassiopeia A taken with 84-foot radio telescope at a wavelength of $10.3 \mathrm{~cm}$.

of the data indicates an equivalent black-body temperature greatly in excess of that previously determined at $3 \mathrm{~cm}$, and which varies with time. Second, a new rather bright source heretofore undetected at $10 \mathrm{~cm}$ has been measured. This source is located in a region of heavy obscuration.

A total of 60 drift curves of Jupiter were made on five days during the period 1958 June 10 to June 28 , using the $48^{\circ}$ time constant. With a beamwidth of 18 minutes of arc, the $48^{8}$ time constant distorted the shape and decreased the amplitude of a drift curve by a factor of about 0.7 ; this factor was calibrated by the relatively bright sources Taurus A, Orion nebula, and Virgo A. Fig. 6 is a drift curve of Virgo A made using the $48^{8}$ time constant and shows the distortion in the shape of the curve. The measured antenna temperature of Virgo $A$ is $7.2^{\circ} \mathrm{K}$, which corresponds to a flux density of $9.6 \times 10^{-25}$ watts $\mathrm{m}^{-2}(\mathrm{c} / \mathrm{s})^{-1}$ for a point source. Virgo $A$ produces no appreciable broadening of the beam using the $4^{8}$ time constant. Fig. 7 is a drift curve of Jupiter made with the $48^{\mathrm{s}}$ time constant. To read the amplitude of the Jupiter drift curves with any accuracy, it was necessary to average them point by point in groups of four. Each drift curve was about $11^{\mathrm{m}}$ in

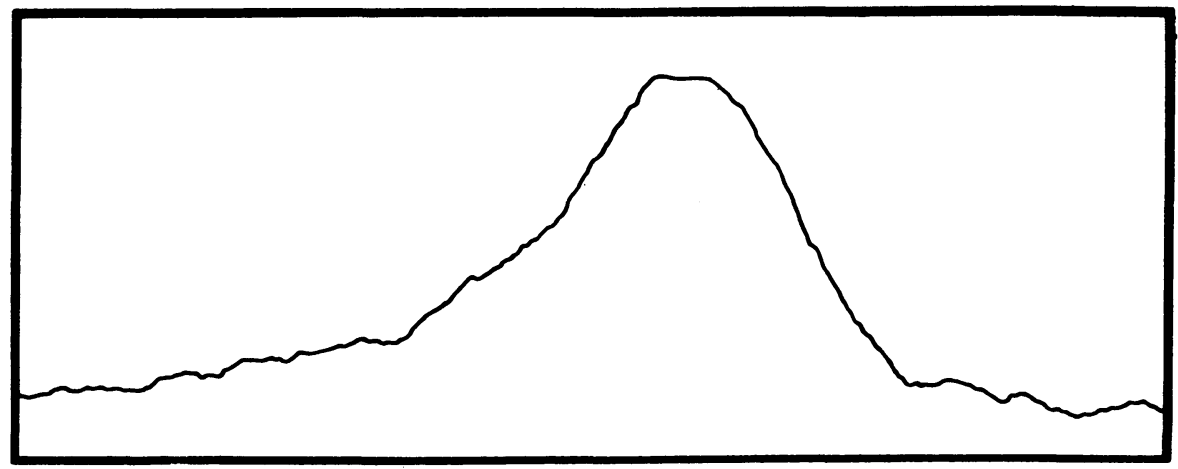

Fig. 6. 1958 June 21 drift curve on Virgo A taken with 84-foot antenna at a wavelength of $10.3 \mathrm{~cm}$. 


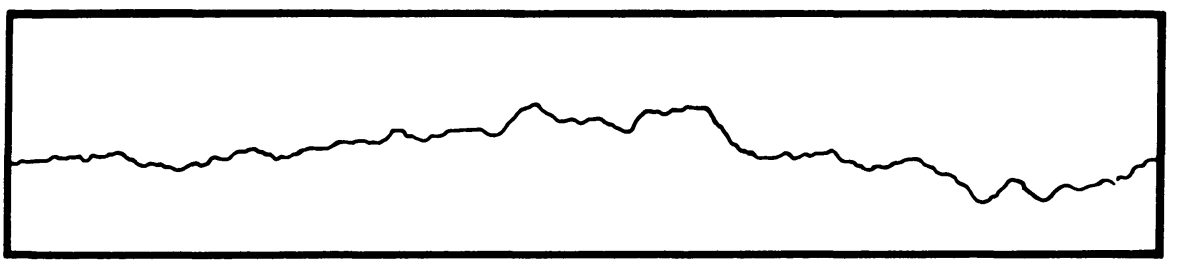

Fig. 7. 1958 June 10 drift curve on Jupiter taken with 84 -foot antenna at a wavelength of $10.3 \mathrm{~cm}$ using $48^{\mathrm{s}}$ time constant.

length, so that one readable average curve was obtained in about $44^{\mathrm{m}}$ of observation. The measured antenna temperatures for these average curves varied from 0.47 to $0.20^{\circ} \mathrm{K}$ with an estimated relative uncertainty of $\pm 0.08{ }^{\circ} \mathrm{K}$. The measured equivalent black-body temperature of Jupiter varied from 860 to $395^{\circ} \mathrm{K}$ with an estimated relative uncertainty of $\pm 160^{\circ} \mathrm{K}$; and the measured flux density varied from $0.62 \times 10^{-25}$ to $0.27 \times 10^{-25}$ watts $\mathrm{m}^{-2}(\mathrm{c} / \mathrm{s})^{-1}$ with an estimated relative uncertainty of $\pm 0.11 \times 10^{-25}$ watts $\mathrm{m}^{-2}(\mathrm{c} / \mathrm{s})^{-1}$. During one night's observation, the equivalent black-body temperature varied from 860 to $390^{\circ} \mathrm{K}$. Attempts to correlate the variations in equivalent black-body temperature with the planet rotation, while slightly suggestive, are by no means conclusive. It is hoped that additional measurements will clarify this point. The average equivalent black-body temperature of all of the $10.3-\mathrm{cm}$ measurements is $580^{\circ} \mathrm{K}$. This is in marked contrast to the equivalent blackbody temperature of $145^{\circ} \mathrm{K}$ measured at $3.15 \mathrm{~cm}[9,10]$, and of $130^{\circ} \mathrm{K}$ in the infrared [11]. This measurement would indicate that the microwave spectrum as well as the long wavelength spectrum of Jupiter may be complex.

Fig. 8 is a drift curve of a new $10-\mathrm{cm}$ source made with the $4^{\text {s }}$ time constant. This source appears to be a compound source made up of a source that is small in angular extent with a measured antenna temperature at 10.3 $\mathrm{cm}$ of $8.7^{\circ} \mathrm{K}$ superimposed on a broad underlying base with a measured antenna temperature of about $1.5^{\circ} \mathrm{K}$ that extends about one degree in both right ascension and declination. Both drift curves and declination scans of this source produce curves with a half-power width of about 19.5 minutes of arc when the contribution of the underlying base is removed, corresponding

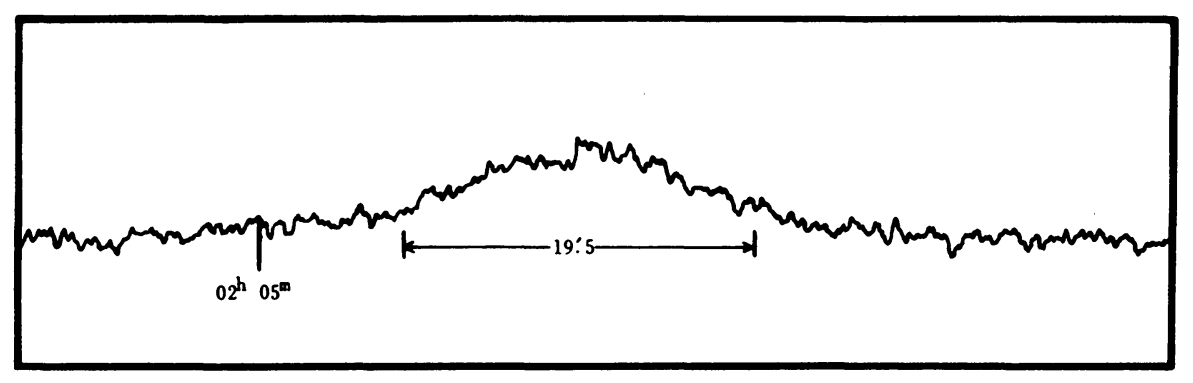

Fig. 8. 1958 April 30 drift curve on source at $i 950$ right ascension $=18^{\mathrm{h}} 44^{\mathrm{m}} 47^{\mathrm{s}}$, declination $=-02^{\circ} 00^{\prime} \cdot 5$ taken with 84 -foot antenna at a wavelength of $10.3 \mathrm{~cm}$. 
to a source with a Gaussian brightness distribution having a half-width of about 7.5 minutes of arc. Assuming a Gaussian antenna beam and a Gaussian source, the measured antenna temperature of $8.7^{\circ} \mathrm{K}$ corresponds to a flux density of about $14 \times 10^{-25}$ watts $\mathrm{m}^{-2}(\mathrm{c} / \mathrm{s})^{-1}$. The measured position of this source is right ascension $=18^{\mathrm{h}} 44^{\mathrm{m}} 47^{\mathrm{s}}$ and declination $=-02^{\circ} 00^{\prime} .5$ (epoch 1950). This position agrees to within 9.0 minutes of arc in right ascension and 1.5 minutes of arc in declination with the position of a source previously measured by Westerhout [12] at a frequency of $1390 \mathrm{Mc} / \mathrm{s}$, for which he measured a flux density of $33.0 \times 10^{-25}$ watts $\mathrm{m}^{-2}(\mathrm{c} / \mathrm{s})^{-1}$. On the basis of these two measurements, this source may have a nonthermal spectrum. As discussed by Westerhout, this source has not been identified; it lies in a direction of heavy obscuration.

\section{REFERENCES}

[1] Dicke, R. H. Rev. Sci. Instrum. 17, 268, 1946.

[2] Drake, F. D., and Ewen, H. I. Proc. I.R.E. 46, 53, 1958.

[3] Haddock, F.T., and McCullough, T. P. Private communication.

[4] Mayer, C. H., McCullough, T. P., and Sloanaker, R. M. Unpublished.

[5] Haddock, F. T., Mayer, C. H., and Sloanaker, R. M. Ap. J. 119, 456, 1954.

[6] Haddock, F. T., Mayer, C. H., and Sloanaker, R. M. Nature, 174, 176, 1954.

[7] Hagen, J. P., McClain, E. F., and Hepburn, N. Proc. I.R.E. 42, 1811, 1954.

[8] Crawford, A. B., Friis, H. T., and Jakes, W. C., Jr. Bell Syst. Tech. J. 35, 1199, 1956.

[9] Mayer, C. H., McCullough, T. P., and Sloanaker, R. M. Proc. I.R.E. 46, 260, 1958.

[10] Mayer, C. H., McCullough, T. P., and Sloanaker, R. M. Ap. J. 127, 11, 1958.

[11] Menzel, D. H., Coblentz, W. W., and Lampland, C. O. Ap. J. 63, 177, 1926.

[12] Westerhout, G. B.A.N. 14, 215, 1958. 